\title{
Evaluating the effects of switching from cigarette smoking to using a heated tobacco product on health effect indicators in healthy subjects: study protocol for a randomized controlled trial
}

\author{
Nik Newland ${ }^{1} \cdot$ Frazer John Lowe $^{1}$ (1) $\cdot$ Oscar Martin Camacho ${ }^{1} \cdot$ Mike McEwan $^{1} \cdot$ Nathan Gale $^{1} \cdot$ James Ebajemito ${ }^{1}$. \\ George Hardie ${ }^{1}$. James Murphy ${ }^{1} \cdot$ Christopher Proctor $^{1}$
}

Received: 16 January 2019 / Accepted: 16 April 2019 / Published online: 2 May 2019

(c) The Author(s) 2019

\begin{abstract}
Tobacco heating products (THPs) are a potentially safer alternative to combustible cigarette smoking. Through continued use, THPs may reduce smoking-related disease risk, whilst maintaining the sensorial experience and nicotine delivery sought by smokers. While literature evidence of the biological effects of THP aerosol exposure is increasing, there remains a knowledge gap with respect to substantiation of THP reduced risk potential in longer term real-life use. This randomized, multi-centre, controlled clinical study will test the hypotheses that following a switch from combustible cigarettes to a THP for 1 year, participants will experience a sustained reduction in exposure to tobacco-related toxicants that will lead to favourable changes in health effect indicators associated with smoking-related disease development. Changes in such indicators will be contextualized against smoking cessation and never-smoker cohorts. Up to 280 participants who do not intend to quit smoking will be randomized to continued combustible smoking (arm A, up to $n=80$ ) or a commercially available THP (arm B $n=200$ ). Furthermore, up to 190 participants with a high intent to quit smoking will undergo smoking cessation (arm D), and 40 never smokers will serve as a control group (arm E). Recruitment numbers were determined to be sufficient to achieve $n=50$ in arms A, B and D, at study end. Enrolment started in March 2018 and the trial is scheduled to be completed in March 2020. Data from this study will be a valuable addition to the growing body of evidence in the field of understanding the individual and public health impact of THPs.

Clinical Trial Registration: https://www.isrctn.com/ISRCTN81075760
\end{abstract}

Keywords Tobacco heating product $\cdot$ PRRP $\cdot$ Cardiovascular disease $\cdot$ Biomarkers $\cdot$ NNAL $\cdot$ Augmentation Index Oxidative stress $\cdot$ Smoking cessation

\section{Introduction}

Cigarette smoking is a well-known cause of human disease [1]. The associated risks are known to correlate with duration of smoking and daily cigarette consumption, and quitting reduces these risks [2,3]. Reducing the negative

Electronic supplementary material The online version of this article (https://doi.org/10.1007/s11739-019-02090-8) contains supplementary material, which is available to authorized users.

Frazer John Lowe

frazer_lowe@bat.com

1 Group Research and Development, British American Tobacco (Investments) Ltd., Regents Park Road, Southampton SO15 8TL, UK health burden of tobacco use is a clear public health priority and has led to a series of regulatory and educational initiatives to persuade people not to smoke $[1,4]$. Despite these efforts, smoking rates in adult populations worldwide remains at $10-40 \%$ in most countries [5], and the World Health Organization (WHO) forecasts that there will be around 1.5 billion tobacco smokers worldwide in 2050 [6]. Therefore, complementing existing tobacco control policies with strategies that attempt to reduce or prevent harm in those who will otherwise continue to smoke is important. Tobacco harm reduction, the substitution of cigarette smoking for potentially reduced-risk nicotine delivery systems, could offer substantial public health gains if widely adopted $[7,8]$. Tobacco researchers and policy experts have long embraced the idea that less harmful sources of nicotine could provide rewarding effects similar to that of cigarettes 
and might entice smokers away from cigarette smoking. This is because nicotine per se (decoupled from tobacco smoke) at doses commonly consumed is relatively safe [9, 10]. Although known for its psychoactive properties [11], nicotine is not a carcinogen [12] and does not contribute to smoking-related diseases [1].

In principle, pharmaceutical nicotine products [also known as nicotine-replacement therapies (NRTs)] that deliver "clean" nicotine (e.g. patches, gums, sprays and inhalers) can also be used for cigarette smoking substitution by virtue of their value in reducing cravings and symptoms of withdrawal in smokers. However, nicotine delivery from NRT products is relatively slow compared with smoking, their pharmacokinetic profiles hardly approximating those of cigarettes with maximum plasma concentrations typically showing a much lower and flatter pharmacokinetic profile compared to cigarettes [13,14]. The deficient pharmacokinetic profiles, poor product appeal, absence of a typical hand to mouth action and satisfaction may explain why many smokers who attempt to quit using NRT products relapse to smoking. Realistic alternatives need to be as readily available as cigarettes are currently, competitively priced, socially acceptable and approved for regular long-term recreational use, while also strongly discouraging use of smoked tobacco [8].

Electronic inhalable vapour products (e-cigarettes) and heated tobacco products (THPs) have received most attention as long-term alternatives to smoking. This study will exclusively investigate a newly marketed THP.

Most THPs heat tobacco sticks, typically to temperatures lower than $350^{\circ} \mathrm{C}$, rather than combusting them. The lack of combustion results in significantly fewer chemical toxicants formed in the aerosol than in cigarette smoke, but nicotine is still delivered. Compared with e-cigarettes, THPs are less well characterised, but assessments of the chemical toxicants found in THP vapour has revealed significant reductions in the levels of many chemical toxicants when compared to those found in conventional cigarette smoke $[15,16]$. In an independent clinical trial measuring $\mathrm{CO}$ levels in exhaled breath of participants using two recently marketed THPs, no $\mathrm{CO}$ elevations could be detected, suggesting that no combustion takes place when using these products [17]. Significant levels of nicotine in THP vapour has also been confirmed $[15,16,18]$.

Several studies have investigated the potential for adverse human health effects of THPs, and in some cases, in comparison with combustible cigarettes. The studies performed to date have predominantly measured chemical emissions [15-21], in vitro endpoints [15, 22-25], in vivo endpoints [26-29] and biomarkers of exposure (BoE) of chemical toxicants found in cigarette smoke in individuals who have switched from smoking to using THPs [30, 31]. In summary, significant reductions in emissions, biological activity and human exposure respectively were rapidly attained and were sustained for at least 1 month of continued use [15, 16, 19]. However, the presence of some emission toxicants, biological activity and elevated BoEs remained, indicating that use of THPs is unlikely to be risk free.

With the substantially altered chemical profile of THP aerosol and the reduced chemical exposure compared with cigarette smoke, long-term negative health effects might also be reduced $[32,33]$. Substantiation of the potential for risk reduction from THP use requires quantification in carefully designed clinical studies [33]. As part of premarketing authorisation applications for novel tobacco products, some regulators require clinical data to identify any potential reduction in individuals' risk relative to continued smoking. To substantiate harm reduction potential, an extensive weight-of-evidence-based scientific dataset is likely needed. We have recently proposed an assessment framework describing the types of studies, which are likely to be needed to generate such a weight-of-evidence [34]. In addition to the datasets already published as part of the framework [35], this study will provide critical information from a real-life setting to determine if the previously characterised reductions in emissions, exposure and in vitro responses, translate into a reduced risk benefit for consumers.

The purpose of this study is twofold:

1. To test the hypothesis that reductions in toxicant delivery from a THP can translate to sustained reduction in human exposure to cigarette smoke toxicants, as assessed by measuring biomarkers of exposure in an ambulatory setting.

2. To test the hypothesis that reduction in exposure to toxicants will cause changes in health effect indicators when smokers switch to using THPs compared with smokers who continue to smoke (CTS), and that these changes are directionally similar to changes seen in smokers who undergo smoking cessation, over a period of $6-12$ months in an ambulatory setting.

\section{Methods}

The full study protocol is provided in the supplementary information, submitted with this manuscript. Due to the removal of arm C from the study, study arm names (A, B, $\mathrm{D}, \mathrm{E})$ in this manuscript have been maintained to align with the protocol and the requirements of GCP. The key aspects of the protocol are summarised below.

\section{Study design and participants}

This will be a multi-centre, unblinded, controlled ambulatory study with a randomised design (Fig. 1). This study will 
Switch

Screening Period (Day -28 to

Day -1)

Exposure Period $\underset{\longrightarrow}{\text { Follow-up Period }}$

$\begin{array}{llllllllllllllll}\text { Study Day } & -28 & 1 & 30 & 60 & 90 & 120 & 150 & 180 & 210 & 240 & 270 & 300 & 330 & 360 & 388\end{array}$

Visit

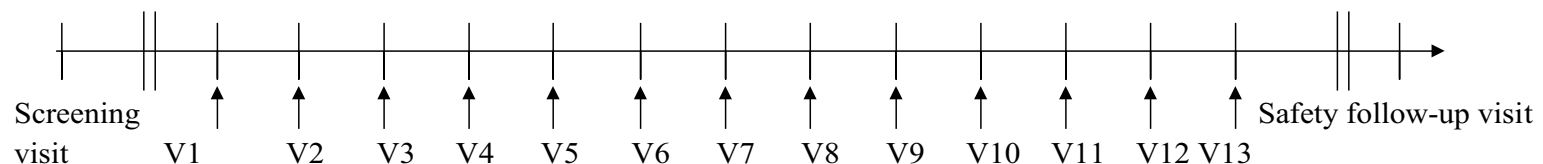

Continue to smoke (Arm A) / THP1.1 (RT) population (Arm B)

\begin{tabular}{|c|c|c|}
\hline Cigarette use & Continue to smoke conventional cigarettes & \\
\hline Cigarette use & THP1.1 (RT) & \\
\hline
\end{tabular}

Intend to quit population (Arm D)

\begin{tabular}{l|r}
\hline Cigarette use & Assisted smoking cessation \\
\hline
\end{tabular}

Never smoked population (Arm E)*

\begin{tabular}{|l|c|c|}
\hline Never smoked & Never smoked & \\
\hline
\end{tabular}

Fig. 1 Study schematic. *Subjects in arm E will only attend screening, visit 1 (day 1), visit 4 (day $90 \pm 3$ days), visit 7 (day $180 \pm 2$ weeks), visit 13 (day 360 ( \pm 2 weeks), and follow-up

be conducted in accordance with consensus ethical principles derived from the Declaration of Helsinki, as well as with the quality standards of Good Clinical Practice. This study has been registered at the following site: https://www. isrctn.com/ISRCTN81075760.

The planned duration is 360 days, and three separate populations will be recruited, to four study arms:

- Current smokers, defined as adult male and female regular smokers of 10-30 non-mentholated commercially manufactured filter cigarettes and/or roll your own cigarettes per day. This population is further split into participants who will continue to smoke their own brand of cigarette for the duration of the study ( $\operatorname{arm~A),~and~}$ participants who will completely switch to THP1.1 (RT) after baseline for the duration of the study (arm B).

- Current smokers intending to quit, defined as adult male and female regular smokers of 10-30 non-mentholated commercially manufactured filter cigarettes and/or roll your own cigarettes per day intending to cease all nonmedicinal nicotine use (arm D).

- Never smokers, defined as having smoked $<100$ cigarettes in their lifetime and none in the 30 days before screening (arm E).

\section{Inclusion criteria}

Full inclusion criteria are listed in the study protocol (see supplementary information), and include

- Age 23-55 years.

- Body mass index (BMI) $17.6-32.0 \mathrm{~kg} / \mathrm{m}^{2}$ and body weight $>50 \mathrm{~kg}$ (men) or $40 \mathrm{~kg}$ (women).

- Good general health (in the opinion of the Principal Investigator or qualified designee).

- Willingness to refrain from consuming alcohol $24 \mathrm{~h}$ before study screening and all study visits.

- Participants in arms A and B must have urine cotinine levels $>200 \mathrm{ng} / \mathrm{mL}$ and exhaled breath CO levels $\geq 7 \mathrm{ppm}$ at screening [36].

- Participants in arms A, and B will have smoked for at least five consecutive years prior to screening.

\section{Exclusion criteria}

Full exclusion criteria are listed in the study protocol (see supplementary information), and include

- Acute illness (e.g. upper respiratory tract infection) requiring treatment within 4 weeks before visit 1 (participants with viral infections that resolved $\geq 2$ weeks before visit 1 will be eligible for inclusion). 
- History of alcoholism or drug/chemical abuse within 24 months before screening or a positive alcohol breath test (confirmed by repeat) at screening or visit 1 .

- Carriers of hepatitis B virus, hepatitis C virus or HIV.

- Use of prescription or over-the-counter bronchodilator medication (e.g. inhaled or oral $\beta$-adrenergic agonists) to treat a chronic condition within the 12 months before visit 1 .

- Use of any medications or substances (other than tobacco) that interfere with the cyclo-oxygenase pathway (e.g. anti-inflammatory drugs including aspirin and ibuprofen) or strong inducers or inhibitors of cytochrome CYP enzymes within 14 days or five half-lives of the drug (whichever is longer) within 14 days before visit 1 .

- Strenuous physical activity within 7 days before screening or visit 1.

- Clinically relevant abnormal findings in medical history or on physical examination (e.g. presence of on-going disease or pathology).

- For participants in arms A, B, and D, use any nicotine or tobacco product other than commercially manufactured filter cigarettes and/or roll your own cigarettes within 14 days of screening.

- In arms A and B, self-reported non-inhalers (smokers who draw smoke from the cigarette into the mouth and throat but who do not inhale).

- In arms A and B, smokers planning to quit in the next 12 months at screening, although smokers who are enrolled are free to quit smoking and withdraw from the study at any time and will be directed to appropriate stop smoking services.

\section{Group allocation and randomisation}

Current smokers will be randomly assigned to a group that will continue to smoke commercially manufactured filter cigarettes and/or roll-your-own cigarettes ( $\operatorname{arm} \mathrm{A})$ or a group that will switch to THP1.1 (RT) (arm B). A randomisation scheme for arms A and B will be provided to the study sites. Randomisation lists will be separated by sex and age categories. Each site will recruit a similar number of men and women which will be randomised by two different age groups: aged 23-40 years and 41-55 years. Random allocation of participants will be monitored throughout the study. If an imbalance is seen that is anticipated to lead to problems when interpreting the data, sites will be requested to try to prioritise the recruitment of participants in the relevant category or categories.

Participants intending to quit will be allocated to arm D and non-smokers to arm E. Although these arms will not be randomised, sites will endeavour to balance these populations during recruitment by sex and the same age groups. Subjects in arm D will determine their smoking cessation strategy with the Investigator or their appropriately trained designee at visit 1 . If necessary, subjects will be provided with nicotine replacement therapy (Invisipatches starting at $25 \mathrm{mg}$ and weaning down \pm an inhalator) or Varenicline, but a combination of this and NRT was not permitted, it was one or the other) or given a prescription for these products. Participants used these products for 12 weeks, or in some cases longer (up to 24 weeks), under the supervision of a qualified general practitioner.

For additional support, subjects will be referred to the following services based on where they live:

- Subjects in England-National Health Service (NHS) quitting support website (https://www.nhs.uk/live-well/ quit-smoking/nhs-stop-smoking-services-help-you-quit), the Smokefree helpline (Tel: 0300123 1044), online advisor support, local stop smoking service, and Smokefree app.

- Subjects in Northern Ireland-Want2Stop quitting support website (www.want2stop.info).

- Subjects in Wales-NHS Wales quitting support website (www.helpmequit.wales) and telephone helpline (0800 085 2219).

Subjects will also be provided with a 24-h site number that they contact for cessation support if required. The Investigator or their appropriately qualified designee will review subject's progress and strategy at each clinic visit.

\section{Compliance}

Product use compliance is a critical part of this study, as failure to fully replace cigarettes with the THP product would reduce or nullify the expected biomarker changes that would be observed if the product is used as indicated. Participants will be instructed of the importance of adhering to their randomised product allocation (arms A and B) or quitting strategy (arm D) and of not smoking (arm E). They will be asked to report any non-compliance via a study diary and will be informed that assessments of adherence will be conducted at selected clinic visits. Analysis of blood $N$-(2-cyanoethyl) valine haemoglobin adducts (CEVal; formed following acrylonitrile exposure) will be conducted on all study participants, as a marker of combusted tobacco exposure. We will use different thresholds for CEVal in ancillary analyses to deduce product use compliance. These thresholds have been calculated based on a previous study where this biomarker was reported for a modified combustible prototype cigarette [37]. 


\section{Study product}

The THP1.1 (RT) is a novel THP that has been designed and manufactured by British American Tobacco (BAT), which is commercially available in Japan, Italy, Canada, Romania, South Korea, Russia, and Switzerland. Product information for THP1.0 (the same heating device, and a similar consumable) is fully described by Eaton et al. [38], and the associated machine-puffed chemical emission data are described by Forster et al. [16]. THP1.1 (RT) has two components: a cylindrical tobacco stick consumable of $83 \mathrm{~mm}$ length and $5.4 \mathrm{~mm}$ diameter [weight $540 \mathrm{mg}$ of which $210 \mathrm{mg}$ is tobacco, and nicotine yield is $0.68 \mathrm{mg}$ per stick (modified Health Canada Intense puffing regime)], and an electronic heating device into which the consumable is inserted for heating before use. The heating device comprises a rechargeable battery, an electrical heating element and electronic hardware that controls device warming, heating temperature and heating period. The device heats the tobacco to a maximum temperature of $240{ }^{\circ} \mathrm{C} \pm 5^{\circ} \mathrm{C}$ [38], and contains safety technology to prevent heating over $260^{\circ} \mathrm{C}$.

To use THP1.1 (RT), the consumable is placed in a port on the top of the THP device, leaving the filter end protruding. The port is accessed by moving a sliding cover (Fig. 2). Once the consumable is inserted, the user pushes and holds an activation button on the THP for $3 \mathrm{~s}$ to start heating process; the THP vibrates briefly and one of a series of four LED lights on the front of the device around the activation button illuminates to indicate this has begun. The remaining lights illuminate, and when the final light is lit, and the device vibrates again, heating is complete and the user many begin puffing. The heating process takes $40 \mathrm{~s}$ and heating at the maximum temperature continues for $210 \mathrm{~s}$, after which the heater shuts down automatically.

\section{Product use}

Participants will be asked to continue smoking their usual brands of cigarette until randomisation (arms A and B) or enrolment (arm D). After randomisation, the participants in arms A and B will be allowed to consume only the assigned products until the final visit on day 360 . Smoking or THP use will be ad libitum but capped at $200 \%$ of self-reported cigarette per day (CPD) consumption at screening. Participants in arm A will be told before the study that they will continue to smoke their usual brand of cigarettes at their own expense. Smokers will be reminded of the risks associated with smoking prior to enrolment, and informed that they are free to voluntarily quit smoking and/or withdraw from the study at any time.

Participants in arm D will be allowed to use only specific NRT and smoking cessation aids from enrolment to day 360 . For participants in arm B, at visit 1, they will be provided with the THP1.1 (RT) and stick consumables equivalent to no more than $150 \%$ of the self-reported number of cigarettes per day (CPD) consumed at screening, with the possibility of more, up to a total of $200 \%$ of original CPD consumption, before visit 2 by visiting the study site. At visits 2-12, product usage will be assessed by return of all empty, partused, and unused packs of THP consumables, and the next batch of product will be supplied at $120 \%$ of usage in the previous period, up to the limit of $200 \%$ of pre-screening

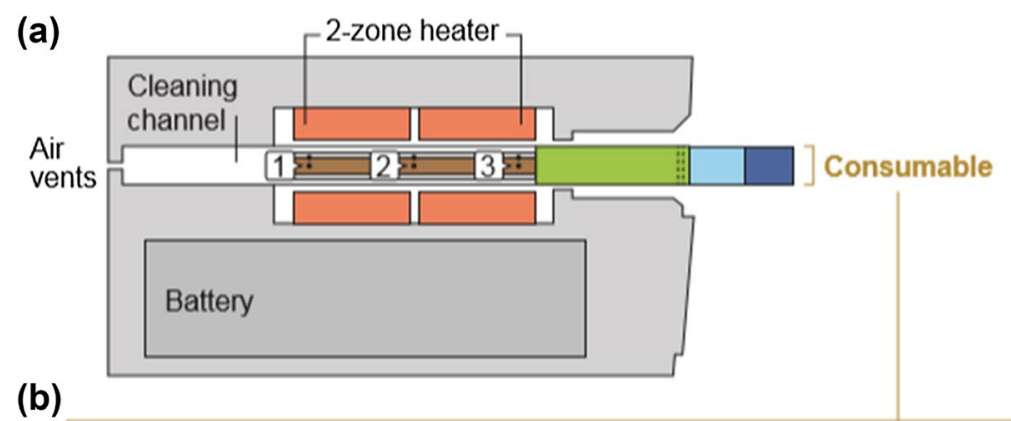

(b)

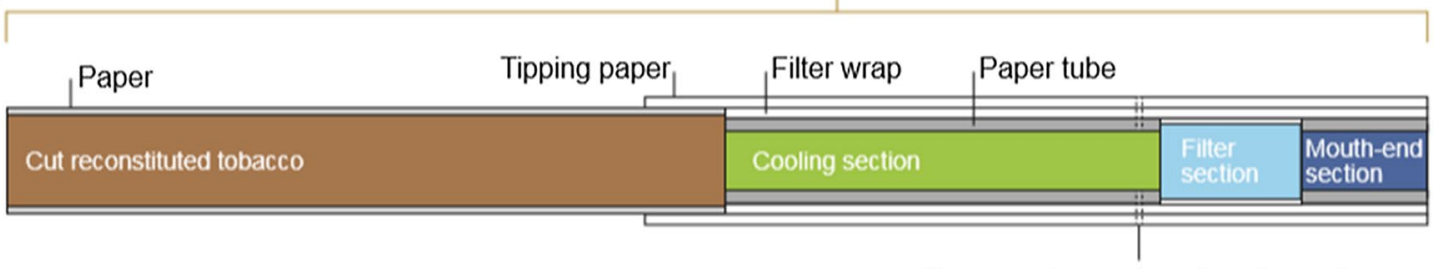

Air expansion and cooling channels

$82 \mathrm{~mm}$ rod length; $5 \mathrm{~mm}$ rod diameter (42 $\mathrm{mm}$ tobacco section length)

Fig. 2 a The THP1.1 (RT) tobacco heating product and b the THP1.1 (RT) consumable 
consumption. At visit 13, as well as all empty, part-used and unused packs of THP consumables, participants will be asked to return the study device, chargers and other accessories supplied for use in this study.

Two devices are supplied to study participants to mitigate the potential for device loss and/or failure/malfunction. Furthermore, a spare device allows the subject to continue use of the product when required, while a depleted battery is recharging. In the event of loss, or a failure of a device (damage or malfunction), subjects are supplied with a telephone contact at the clinic, who will immediately arrange a replacement device.

\section{Study objectives and endpoints}

The primary objective of the study is

- To quantitatively assess differences in primary study endpoints at 90, 180, and 360 days between subjects who continue to smoke conventional cigarettes and subjects who switch to a THP.

Secondary objectives of the study are as follows:

- To quantitatively assess differences in secondary study endpoints between subjects who continue to smoke conventional cigarettes and subjects who switch to a THP.

- To assess differences in all study endpoints between subjects who switch to a THP and subjects in the assisted smoking cessation arm.

- To assess the differences in all study endpoints between subjects who switch to a THP or undertake assisted smoking cessation, and subjects who have never smoked.

Table 1 Analysis schedule

\begin{tabular}{lll}
\hline $\begin{array}{l}\text { Analysis } \\
\text { schedule }\end{array}$ & Study visit & Study timepoint \\
\hline 1 & All visits & All days \\
2 & $1,2,3,4,7,10,13$ & $0,30,60,90,180,270,360$ \\
3 & $1,4,7,10,13$ & $0,90,180,270,360$ \\
\hline
\end{tabular}

- To monitor the safety profile of subjects using THPs and combustible cigarettes, and subjects in the smoking cessation and never-smoker arms.

Exploratory objectives are as follows:

- To quantitatively assess the time required to observe changes in selected primary and secondary endpoints following a switch from conventional cigarettes to a THP or assisted cessation.

- To quantitatively assess differences in the exploratory endpoints between subjects who continue to smoke conventional cigarettes, subjects who switch to a THP, subjects in the cessation arm, and subjects who have never smoked (to be reported separately).

- To investigate the profile of selected primary and secondary endpoints over the course of the study for each study arm.

The endpoint analysis schedule is presented in Table 1, and the study endpoints are shown in Tables 2 and 3.

Additional exploratory biomarker endpoints of interest will be body fat, tetrahydrobiopterin/dihydrobiopterin (BH4/ $\mathrm{BH} 2$ ) ratio in plasma, untargeted and targeted transcriptomics (assessed in nasal epithelial cells and white blood cells), targeted lipoprotein levels (by nuclear magnetic resonance; NMR) in serum and targeted metabolomics in serum.

A detailed schedule of assessments can be found in the supplementary information.

\section{Statistical power considerations}

Statistical power calculations based upon the primary endpoint that required the largest sample size to observe change [Augmentation Index (AIx)] determined that 50 participants completing the study in arms A, B, and D will provide sufficient power to allow multiple between-arm comparisons between the test and control products. Specifically, the power calculation was based on the number of participants required to perform a contrast based on the $F$ statistic, with $90 \%$ power between the arm B (THP) and arm A (continued

Table 2 Primary endpoint biomarkers

\begin{tabular}{|c|c|c|c|c|}
\hline Biomarker & Abbreviation & Matrix & Indication & $\begin{array}{l}\text { Analysis } \\
\text { Schedule }\end{array}$ \\
\hline $\begin{array}{l}\text { Total 4-(methylnitrosa- } \\
\text { mino)-1-(3- pyridyl)-1-bu- } \\
\text { tanol }\end{array}$ & Total NNAL & Urine $(24 \mathrm{~h})^{\mathrm{a}}$ & $\begin{array}{l}\text { Metabolite of the smoke toxicant } \\
\text { 4-(methylnitrosamino)-1-(3-pyridyl)-1-butanone } \\
\text { (NNK) }\end{array}$ & 2 \\
\hline 8-epi-Prostaglandin $\mathrm{F}_{2 \alpha}$ type III & 8-Epi-PGF $2 \alpha$ type III & Urine $(24 \mathrm{~h})^{\mathrm{a}}$ & Marker of oxidative stress & 2 \\
\hline Augmentation Index & AIx & Physiological measure & $\begin{array}{l}\text { Marker of arterial stiffness and hence an indicator for } \\
\text { cardiovascular risk }\end{array}$ & 2 \\
\hline
\end{tabular}

\footnotetext{
${ }^{\mathrm{a} A m b u l a t o r y}$ collection
} 


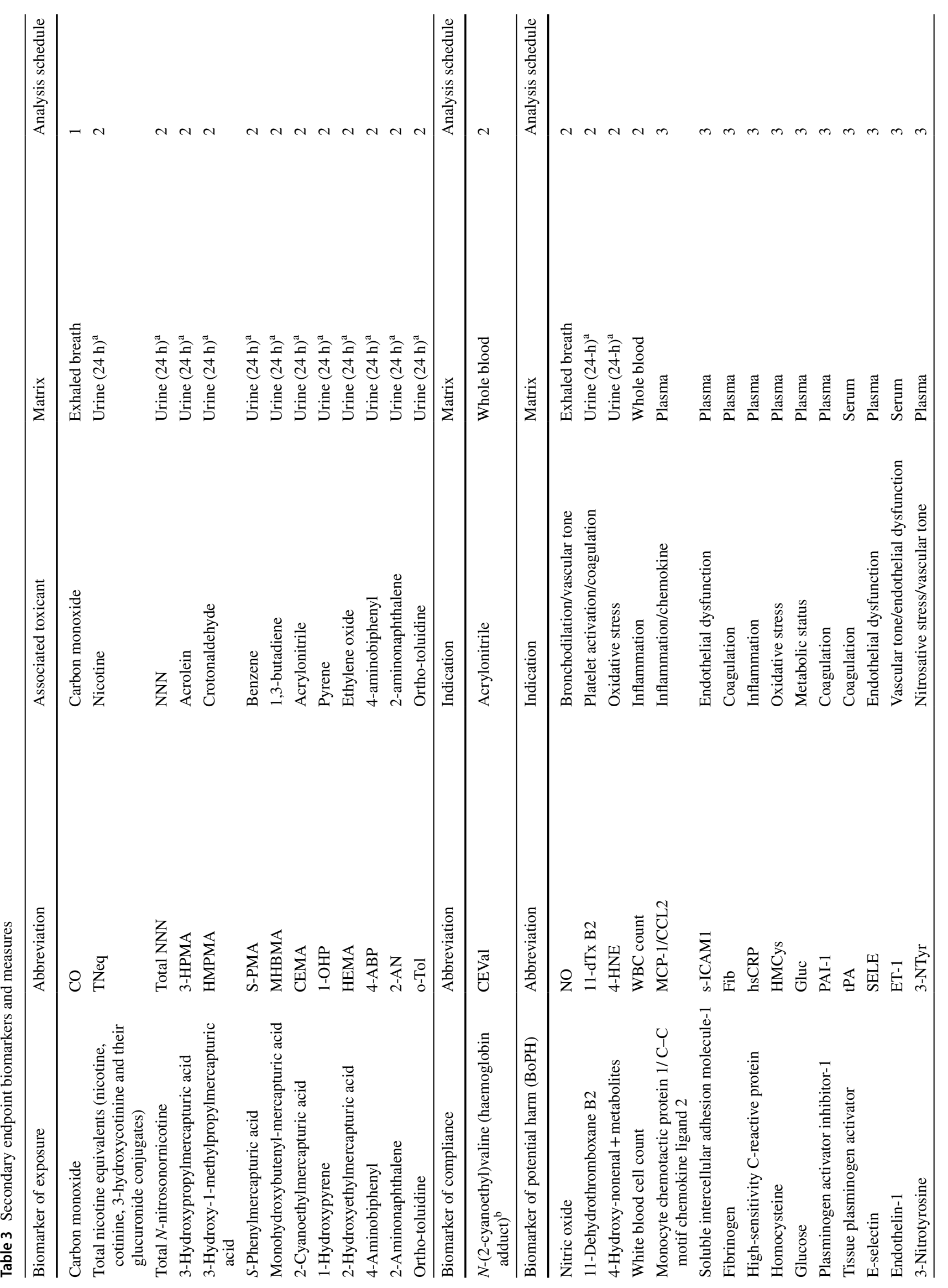




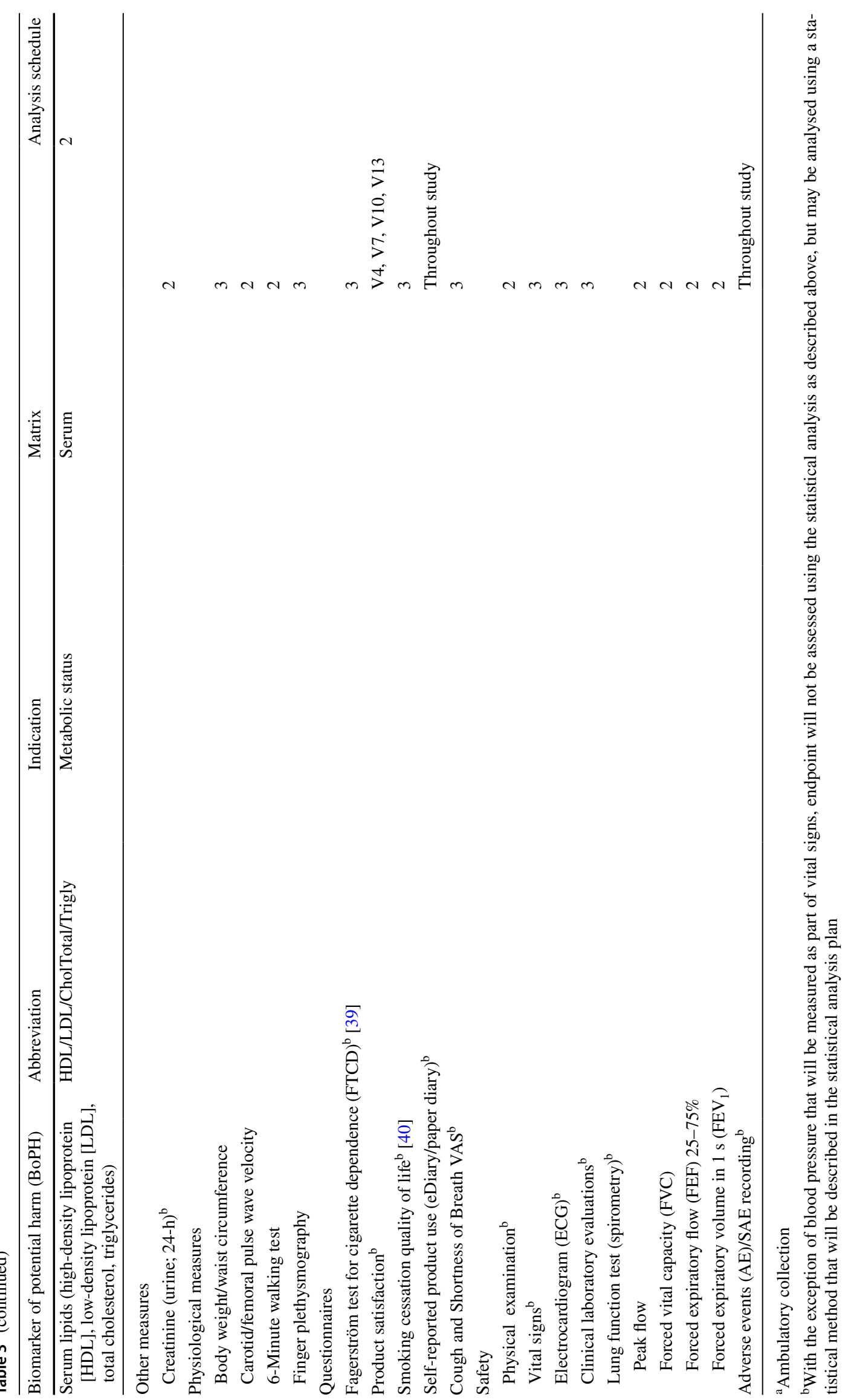


smoking) at day 360 . The sample size was determined to be adequate based on AIx with expected means of $25.7 \%$ and $17.5 \%$ for smoker and THP arms, respectively, and a common standard deviation of $12.4 \%$ [41]. This calculation assumes an $80 \%$ change from baseline with the THP, in line with that observed in participants quitting smoking and an alpha level of 0.0451 , adjusted for timepoint multiplicity using O'Brien-Fleming sequential approach [42]. Thus, recruitment targets are 80 participants to arm A, 200 to arm B and 190 to arm D. In arm E, we have determined a sample size of 30, empirically with no formal statistical analysis, as this cohort's biomarker profile is expected to be stable throughout the study.

\section{Statistical analysis of primary and secondary objectives}

The primary objective will be examined by computing levels of biomarkers at each timepoint, i.e. baseline, 90, 180, and 360 days. These data will be compared between the THP $\operatorname{arm}(\operatorname{arm} B)$ and the continued smoking $\operatorname{arm}(\operatorname{arm} \mathrm{A})$ using specific contrast tests from statistical models adjusted for baseline measurements. Data will be examined and may be transformed to ensure that any assumptions associated with statistical tests or models are obeyed. Alpha level across timepoints has been adjusted using the O'Brien-Fleming approach, with 0.0471 overall available alpha at day 360 , 0.0151 at day 180 , and 0.0006 at day 90 [42]. The significance level has been allocated at each primary endpoint based on likelihood of success to detect a significant change in biomarker levels. At day 360, statistical comparisons between THP and control will be performed at $\alpha=0.0469$ for AIx. The remainder alpha will be distributed equally $(\alpha=0.0001)$ for the other two primary endpoints. At day 90 , only changes in BoE are expected; therefore, only total NNAL will be statistically assessed with $\alpha=0.0006$. For the statistical analysis performed at day 180, an overall $\alpha$ level of 0.0151 will be equally distributed between the three primary endpoints $(0.00503)$. If any endpoint were to be significant at day 90 or 180, it will not be statistically assessed at day $180 \mathrm{and} /$ or 360, as appropriate, and its assigned alpha level will be equally distributed between the remaining primary endpoints.

Similarly, biomarker measures in the secondary objectives will be examined by computing levels of biomarkers at each timepoint. These data will be compared between the THP and the main control arm using specific contrast tests from statistical models adjusted for baseline measurements. Statistical comparisons for secondary endpoints will be only performed if any of the primary endpoints is significant and using the alpha level released by primary endpoints. If the statistical comparison for total NNAL is significant at day 90 , only the secondary BoE will be analysed at day 90 using a 0.0006 significance level. Multiplicity adjustment for family-wise error of secondary endpoints will be performed using Holm's method. If any secondary endpoint were to be significant at day 90 or 180 , it will not be statistically assessed at day 180 and/or 360, as appropriate.

Product use compliance is a critical part of this study, as failure to fully replace cigarettes with the THP product would reduce or cancel the expected biomarker changes that would be observed if the product is used as indicated. To aid compliance assessment we will use a haemoglobin adduct of acrylonitrile; $N$-(2-cyanoethyl)valine (CEVal). Acrylonitrile is below the detection limit in the THP product emissions but can be found in cigarette smoke. We will use different thresholds for CEVal in ancillary analyses to deduce product use compliance. These thresholds have been calculated based on a previous study where this biomarker was reported for a modified combustible prototype cigarette [43].

The interim analysis on day 90 will be performed on a subset of subjects who were enrolled in the study on or before the day that the 42nd subject was enrolled on to $\operatorname{arm~A}$, and were still enrolled in the study at day 90 . This was chosen to ensure that 30 subjects on arm A were still enrolled on the study at day 90 to give sufficient power to detect the statistical difference between the two arms for total NNAL.

All safety data will be summarised for all safety parameters based on the safety population, stratified by study arm.

\section{Expected results}

Enrolment started in March 2018 and the trial is ongoing. The results of this study are expected in 2020 .

\section{Discussion}

Scientific studies on next-generation tobacco and nicotine products are increasing in number recently; however, there is still a substantial gap in knowledge with respect to the longer term health effects associated with their use. While there are now numerous studies assessing the biological effects of electronic cigarettes, datasets relating to THPs are still very limited, and more information is needed to inform consumers, public health and regulation alike. This study will be the first to our knowledge to longitudinally investigate the exposure and health effects associated with THP use over a 12-month period and, furthermore, compare those data to that of assisted smoking cessation and continued combustible product use over the same period of time. The ambulatory design of this multi-centre study will facilitate observation of exposure and health effects associated with real-life use of the THP, hence a key critical challenge to 
the success of the study will be participant compliance with exclusive product use.

\section{Study participant compliance}

Compliance to the study protocol is critical to achieve the scientific objectives of the study. Studies which utilise clinical confinement are somewhat easier to monitor in terms of compliance, as clinic staff are constantly on hand to ensure participants adhere to protocol. However, in an ambulatory setting where participants continue their everyday lives away from the clinic, controls and monitoring tools must be used to ensure, to the greatest degree possible, that participants adhere to the protocol. While some areas of compliance are easily monitored, and breaches easily detectable (e.g. attendance of study visits), others are less so, and a great deal of reliance is placed upon self-reported information from study participants, which may, or may not be $100 \%$ complete or accurate.

A key area of compliance for this study is the adherence to exclusive product use and smoking cessation (for the relevant groups) throughout the full study duration. With respect to smoking cessation, self-reported diaries are usually employed to track cigarette use throughout a trial and can provide insight into whether or not a participant who is on a smoking cessation protocol is continuing to smoke cigarettes. However, participants in clinical trials such as this often fail to fill out self-reported diaries correctly, accurately or even at all. Enthusiasm and compliance at the very start of such a trial are likely to be high. But within the early stages of the study, enthusiasm and compliance have been shown to wane significantly, along with effective selfreporting [44]. Clinical staff who interact with participants at each study visit are limited in their ability to monitor such compliance. Furthermore, they are especially hampered when separate study visits span several months/years, as the opportunity to review diary compliance and subsequently encourage non-compliant participants to return to compliance is limited by the visit schedule. Often, the lack of information in self-reported diaries from missed entries may undermine confidence in the data, and hence the overall study conclusions. Therefore, utilization of improved and more objective measures of adherence is needed to protect the accuracy of the data and, in turn, the overall conclusions of the study. In this study, a combination of different strategies will be employed to ensure the highest level of compliance.

\section{Electronic diaries}

In this study, participants will self-report product use at each study visit, via the use of diaries. To mitigate against missed information, we have provided participants with an electronic diary application which can be accessed via a mobile phone or tablet. The electronic diary will prompt users to complete the diary and inform the clinic staff when entries have been missed. Clinic staff will then be able to make follow-up calls to provide support and assistance if required.

2. Product counting

A measure of overall compliance to product use will be evaluated by counting all empty, part-used, and unused packs of THP consumables returned to investigators.

3. Biomarker of compliance

Given that elimination half-lives of most BoEs are relatively rapid (days/weeks) and are under investigation in this study, further biomarkers are needed to monitor product compliance over a longer period of time. For this, we will use the blood-borne haemoglobin adduct, CEVal, which is formed following exposure to acrylonitrile, a major tobacco smoke constituent. The approximate half-life of this biomarker is 120 days, as it mirrors the life cycle of red blood cells in vivo [37].

\section{Study design}

The protocol incorporates a number of innovative approaches that contribute to the specific uniqueness and quality of the study.

Participants in this study will be a minimum of 23 years of age, which is 18 years (the legal age to obtain tobacco products in the United Kingdom) plus a smoking history of at least 5 years. The study will also ensure that a wide selection of ages is investigated in the study by recruiting individuals between 23 and 55 years old. Given that some of the health effect indicators (such as AIx) are known to correlate with age [45], randomisation has been set to ensure that age bias is minimised, and an equal balance of younger and older participants is recruited. Furthermore, individuals older than 55 years of age are excluded due to the increased likelihood of the presence of clinical or sub-clinical disease.

Smokers are defined as "adult male and female regular smokers of 10-30 non-mentholated commercially manufactured filter cigarettes and/or roll your own cigarettes per day". These limits have been set due to the presence of significant differences in cohorts of smokers who smoke less than and greater than 10 cigarettes per day for some of the biochemical endpoints.

To investigate the effects of smoking cessation over 12 months, a population of subjects who are intending to quit smoking will be recruited, thus maximizing the proportion of these subjects completing the study. The inclusion of a smoking cessation arm in this study is critical to provide context to any favourable changes observed when participants switch from smoking to the THP. Furthermore, the cessation arm will also demonstrate the best possible outcome within the timeframe of the study, in terms of the 
study endpoints, and the extent to which they return to levels observed in never-smokers.

Where BoEs have been shown to change relatively rapidly (days/weeks), health effect indictors such as BoPHs require more time (weeks/months) to observe meaningful change following smoking cessation [46]. Thus, the study period of 12 months is expected to provide sufficient time to allow smoking cessation-responsive BoPHs to change in a favourable manner. Furthermore, as several BoPHs have been shown to change in shorter periods of time, the length of this study will also allow the opportunity to observe if any favourable changes are sustained, and whether or not they return to levels observed in never-smokers.

\section{Health effect indicators}

The health effect indicators selected for this study include a combination of biofluid and physiological analysis for biomarkers of potential harm $(\mathrm{BoPH})$ and health assessment questionnaires. The BoPH included in this study were selected based upon their propensity to change in healthy individuals following smoking cessation.

The BoPH selected for evaluation include a suite of biomarkers that are traditionally associated with cardiovascular/ respiratory disease risk (e.g. HDL/LDL cholesterol, fibrinogen and spirometry assessments) [46]. To complement these, numerous biomarkers have been selected which indicate change in earlier mechanisms involved in the development of cardiovascular and respiratory disease. For example, hypertension is a known risk factor for future myocardial infarction and stroke [47], and the measurement of systolic and diastolic blood pressure over time, can provide insight into the likelihood of the future development of hypertension [47]. To augment insight into the factors modulating changes in blood pressure, a suite of BoPHs has been included to monitor cause-effect relationships at the molecular, cellular and tissue levels of biological hierarchy, which are reported to be mechanistically involved in the modulation of blood pressure over time. Such BoPH provide insight into the level of vascular oxidative/nitrosative stress (a key driver of smoking-related vascular disease) [48], nitric oxide bioavailability (a key mediator of vasodilation and vascular tone) [49] and arterial stiffness [50]. Further BoPH will be measured which relate to platelet activation, coagulation, inflammation, cell adhesion, endothelial dysfunction and metabolic status. Finally, 'omic approaches' will be utilised to assess holistic changes over time in biological samples of respiratory and circulatory origin, and health-related questionnaires will be utilised to provide insight into more subjective perceptions of health and quality of life over time.

In concert, these tools will provide much needed insight into the early processes involved in the development of smoking-related diseases, and better characterise the health-related improvements associated with smoking cessation. With the BoPH profiles of continued smoking, smoking cessation and never smoking characterised, the THP 1.1 (RT) data will be analysed to determine if its exclusive use facilitates a BoPH profile similar to that of smoking cessation, or not.

\section{Study limitations}

Compliance in a study substantiating risk reduction when switching from combustible cigarettes to THPs is paramount. The appeal and the overall sensorial experience of the product under investigation could be a limiting factor to this extent. Exclusive use of the THP product and 100\% adherence to smoking cessation protocols are notoriously difficult to both monitor and achieve. Long-term acceptability and tolerability data for the THP are not yet available and, therefore, it is possible that participants using the THP will continue to use combustible cigarettes to varying degrees in parallel. While the compliance tools that are included in the study will certainly help to identify such "dual-use" participants, they will not be perfect.

The study participants will be recruited from the UK population alone; therefore, the conclusions of the study may not be reflective of populations from other geographical locations and cultures. Furthermore, the study population will consist of healthy adult volunteers aged 23-55 years of age, hence the data may not be reflective of the potential effects of THP use in populations with clinical disease or other vulnerable populations, or older and younger individuals.

Although the study period of 12 months is expected to provide sufficient time to detect smoking cessation-responsive $\mathrm{BoPH}$ changes, it is still possible that for some of them, a much longer follow-up period might be required.

Generalisation of the study data to other THPs should be undertaken with extreme caution. While THPs share a common feature in heating tobacco, rather than burning it, the product heating devices are often designed very differently within the category. Furthermore, the consumables also share significant differences in physical characteristics and ingredients. Taken together, such differences have the potential to yield very different chemical emission profiles, use behaviours and ultimately, biological effects and disease risk. That said, there is potential for bioequivalence exercises to extrapolate the data to similar products where it is appropriate to do so (i.e. under suitable criteria demonstrating that the original and variant products are sufficiently similar to conduct such an exercise, and for a specific regulatory purpose). Such exercises are likely to require additional data packages to support product similarity. 


\section{Summary}

Where reductions in chemical emissions and human toxicant exposure have already been demonstrated with THP use in the short-term, the longer-term effects of THP use in the general population remain unknown. Data from this study will be a valuable addition to the growing body of evidence in the field of understanding the public health impact of THPs. It will generate a pioneering dataset from which the public health community, regulators and consumers can gain insight into whether or not THPs provide a viable and safer alternative to combustible cigarette smoking in adult populations who chose to smoke, or are unable to quit smoking.

Acknowledgements The authors greatly appreciate the efforts, expertise and professionalism of Covance (Leeds, UK), Celerion (Belfast, UK), Richmond Pharmacology (London, UK) and Simbec Orion (Merthyr Tydfil, UK) for their management and conduct of the clinical phase of the study.

\section{Compliance with ethical standards}

Conflict of interest All authors are current employees and stockholders of British American Tobacco (Investments) Ltd, which is the sponsor and funding source of this study. British American Tobacco (Investments) Ltd is the manufacturer and holder of the intellectual property rights of the investigational product used in this study.

Statement of human and animal rights All procedures performed in studies involving human participants were in accordance with the ethical standards of the national research committee (NHS Health Research Authority, Wales Research Ethics Committee 2 (reference number: 17/ WA/0212)) and with the 1964 Helsinki declaration and its later amendments. This article does not contain any studies with animals performed by any of the authors.

Informed consent Informed consent was obtained from all individual participants included in the study.

Open Access This article is distributed under the terms of the Creative Commons Attribution 4.0 International License (http://creativeco mmons.org/licenses/by/4.0/), which permits unrestricted use, distribution, and reproduction in any medium, provided you give appropriate credit to the original author(s) and the source, provide a link to the Creative Commons license, and indicate if changes were made.

\section{References}

1. US Department of Health and Human Services (2014) The health consequences of smoking: 50 years of progress: a report of the surgeon general. US Department of Health and Human Services, Centers for Disease Control and Prevention, National Center for Chronic Disease Prevention and Health Promotion, Office on Smoking and Health, Atlanta. https://www.surgeongeneral.gov/ library/reports/50-years-of-progress/. Accessed 11 Jan 2019
2. US Department of Health and Human Services (1990) The health benefits of smoking cessation. US Department of Health and Human Services. Public Health Service. Centers for Disease Control. Center for Chronic Disease Prevention and Health Promotion. Office on Smoking and Health. DHHS Publication No.(CDC) 90-8416. https://profiles.nlm.nih.gov/ps/access/NNBBCV.pdf. Accessed 11 Jan 2019

3. Doll R, Peto R, Wheatley K, Gray R, Sutherland I (1994) Mortality in relation to smoking: 40 years' observations on male British doctors. BMJ 309(6959):901-911

4. World Health Organization (2011) WHO report on the global tobacco epidemic 2011: Warning about the dangers of tobacco in (World Health Organization, 2011). https://www.who.int/tobacco/ global_report/2011/en/. Accessed 11 Jan 2019

5. Prevalence of Tobacco Smoking (2019) World health Organization. https://www.who.int/gho/tobacco/use/en/. Accessed 11 Jan 2019

6. World Health Organization. https://www.who.int/tobacco/en/atlas 38.pdf. Accessed 11 Jan 2019

7. Polosa R, Rodu B, Caponnetto P, Maglia M, Raciti C (2013) A fresh look at tobacco harm reduction: the case for the electronic cigarette. Harm Reduct J 4(10):19. https://doi. org/10.1186/1477-7517-10-19

8. Rodu B (2011) The scientific foundation for tobacco harm reduction, 2006-2011. Harm Reduct J 8:19. https://doi. org/10.1186/1477-7517-8-19

9. Greenland S, Satterfield M, Lanes S (1998) A meta-analysis to assess the incidence of adverse effects associated with the transdermal nicotine patch. Drug Saf 18(4):297-308

10. Lee PN (2013) The effect on health of switching from cigarettes to snus-a review. Regul Toxicol Pharmacol 66(1):1-5. https:// doi.org/10.1016/j.yrtph.2013.02.010

11. Polosa R, Benowitz NL (2011) Treatment of nicotine addiction: present therapeutic options and pipeline developments. Trends Pharmacol Sci 32(5):281-289. https://doi.org/10.1016/j. tips.2010.12.008

12. World Health Organization International Agency for Research on Cancer (2019) IARC monographs on the evaluation of carcinogenic risks to humans. Volume 83, tobacco smoke and involuntary smoking 2004. International Agency for Research on Cancer, Geneva. https://monographs.iarc.fr/ENG/Monographs/vol83 /mono83.pdf. Accessed 11 Jan 2019

13. Lunell E, Molander L, Ekberg K, Wahren J (2000) Site of nicotine absorption from a vapour inhaler-comparison with cigarette smoking. Eur J Clin Pharmacol 55:737-741

14. Digard H, Proctor C, Kulasekaran A, Malmqvist U, Richter A (2013) Determination of nicotine absorption from multiple tobacco products and nicotine gum. Nicotine Tob Res 15(1):255261. https://doi.org/10.1093/ntr/nts123

15. Schaller JP, Keller D, Poget L, Pratte P, Kaelin E, McHugh D, Cudazzo G, Smart D, Tricker AR, Gautier L, Yerly M, Reis Pires R, Le Bouhellec S, Ghosh D, Hofer I, Garcia E, Vanscheeuwijck P, Maeder S (2016) Evaluation of the tobacco heating system 2.2. Part 2: chemical composition, genotoxicity, cytotoxicity, and physical properties of the aerosol. Regul Toxicol Pharmacol 81(Suppl 2):S27-S47. https://doi.org/10.1016/j.yrtph.2016.10.001

16. Forster M, Fiebelkorn S, Yurteri C, Mariner D, Liu C, Wright C et al (2018) Assessment of novel tobacco heating product THP1.0. Part 3: comprehensive chemical characterisation of harmful and potentially harmful aerosol emissions. Regul Toxicol Pharmacol 93:14-33. https://doi.org/10.1016/j.yrtph.2017.10.006

17. Caponnetto $\mathrm{P}$, Maglia M, Prosperini G, Busà B, Polosa R (2018) Carbon monoxide levels after inhalation from new generation heated tobacco products. Respir Res 19(1):164. https://doi. org/10.1186/s12931-018-0867-z 
18. Farsalinos KE, Yannovits N, Sarri T, Voudris V, Poulas K (2018) Nicotine delivery to the aerosol of a heat-not-burn tobacco product: comparison with a tobacco cigarette and e-cigarettes. Nicotine Tob Res 20(8):1004-1009. https://doi.org/10.1093/ntr/ntx138

19. Jaccard G, Tafin Djoko D, Moennikes O, Jeannet C, Kondylis A, Belushkin M (2017) Comparative assessment of HPHC yields in the tobacco heating system THS2.2 and commercial cigarettes. Regul Toxicol Pharmacol 90:1-8. https://doi.org/10.1016/j.yrtph 2017.08.006

20. Farsalinos KE, Yannovits N, Sarri T, Voudris V, Poulas K, Leischow SJ (2018) Carbonyl emissions from a novel heated tobacco product (IQOS): comparison with an e-cigarette and a tobacco cigarette. Addiction 113(11):2099-2106. https://doi.org/10.1111/ add. 14365

21. Mallock N, Böss L, Burk R, Danziger M, Welsch T, Hahn H, Trieu HL, Hahn J, Pieper E, Henkler-Stephani F, Hutzler C, Luch A (2018) Levels of selected analytes in the emissions of "heat not burn" tobacco products that are relevant to assess human health risks. Arch Toxicol 92(6):2145-2149. https://doi.org/10.1007/ s00204-018-2215-y

22. Nabavizadeh P, Liu J, Havel CM, Ibrahim S, Derakhshandeh R, Jacob Iii P, Springer ML (2018) Vascular endothelial function is impaired by aerosol from a single IQOS HeatStick to the same extent as by cigarette smoke. Tob Control 27(Suppl 1):s13-s19. https://doi.org/10.1136/tobaccocontrol-2018-054325

23. Zanetti F, Titz B, Sewer A, Lo Sasso G, Scotti E, Schlage WK, Mathis C, Leroy P, Majeed S, Torres LO, Keppler BR, Elamin A, Trivedi K, Guedj E, Martin F, Frentzel S, Ivanov NV, Peitsch MC, Hoeng J (2017) Comparative systems toxicology analysis of cigarette smoke and aerosol from a candidate modified risk tobacco product in organotypic human gingival epithelial cultures: a 3-day repeated exposure study. Food Chem Toxicol 101:15-35. https://doi.org/10.1016/j.fct.2016.12.027

24. Breheny D, Adamson J, Azzopardi D, Baxter A, Bishop E, Carr T, Crooks I, Hewitt K, Jaunky T, Larard S, Lowe F, Oke O, Taylor M, Santopietro S, Thorne D, Zainuddin B, Gaça M, Liu C, Murphy J, Proctor C (2017) A novel hybrid tobacco product that delivers a tobacco flavour note with vapour aerosol (part 2): in vitro biological assessment and comparison with different tobacco-heating products. Food Chem Toxicol 106(Pt A):533-546. https://doi.org/10.1016/j.fct.2017.05.023

25. Taylor M, Thorne D, Carr T, Breheny D, Walker P, Proctor C, Gaça M (2018) Assessment of novel tobacco heating product THP1.0. Part 6: a comparative in vitro study using contemporary screening approaches. Regul Toxicol Pharmacol 93:62-70. https://doi.org/10.1016/j.yrtph.2017.08.016

26. Phillips BW, Schlage WK, Titz B, Kogel U, Sciuscio D, Martin F, Leroy P, Vuillaume G, Krishnan S, Lee T, Veljkovic E, Elamin A, Merg C, Ivanov NV, Peitsch MC, Hoeng J, Vanscheeuwijck P (2018) A 90-day OECD TG 413 rat inhalation study with systems toxicology endpoints demonstrates reduced exposure effects of the aerosol from the carbon heated tobacco product version 1.2 (CHTP1.2) compared with cigarette smoke. I. Inhalation exposure, clinical pathology and histopathology. Food Chem Toxicol 116(Pt B):388-413. https://doi. org/10.1016/j.fct.2018.04.015

27. Tsuji H, Okubo C, Fujimoto H, Fukuda I, Nishino T, Lee KM, Yoshimura $\mathrm{H}$ (2014) Comparison of dermal tumor promotion activity of cigarette smoke condensate from prototype (heated) cigarette and reference (combusted) cigarette in SENCAR mice. Food Chem Toxicol 72:187-194. https://doi.org/10.1016/j. fct.2014.07.008

28. Sewer A, Kogel U, Talikka M, Wong ET, Martin F, Xiang Y, Guedj E, Ivanov NV, Hoeng J, Peitsch MC (2016) Evaluation of the tobacco heating system 2.2 (THS2.2). Part 5: microRNA expression from a 90-day rat inhalation study indicates that exposure to THS2.2 aerosol causes reduced effects on lung tissue compared with cigarette smoke. Regul Toxicol Pharmacol 81(Suppl 2):S82-S92. https://doi.org/10.1016/j.yrtph.2016.11.018

29. Oviedo A, Lebrun S, Kogel U, Ho J, Tan WT, Titz B, Leroy P, Vuillaume G, Bera M, Martin F, Rodrigo G, Esposito M, Dempsey R, Ivanov NV, Hoeng J, Peitsch MC, Vanscheeuwijck P (2016) Evaluation of the tobacco heating system 2.2. Part 6: 90-day OECD 413 rat inhalation study with systems toxicology endpoints demonstrates reduced exposure effects of a mentholated version compared with mentholated and non-mentholated cigarette smoke. Regul Toxicol Pharmacol 81(Suppl 2):S93-S122. https://doi.org/10.1016/j.yrtph.2016.11.004

30. Gale N, McEwan M, Eldridge AC, Fearon IM, Sherwood N, Bowen E, McDermott S, Holmes E, Hedge A, Hossack S, Wakenshaw L, Glew J, Camacho OM, Errington G, McAughey J, Murphy J, Liu C, Proctor CJ (2018) Changes in biomarkers of exposure on switching from a conventional cigarette to tobacco heating products: a randomized, controlled study in healthy Japanese subjects. Nicotine Tob Res. https://doi.org/10.1093/ntr/nty10 4 (Epub ahead of print (https://www.ncbi.nlm.nih.gov/pubme $\mathrm{d} / 29912406))$

31. Martin Leroy C, Jarus-Dziedzic K, Ancerewicz J, Lindner D, Kulesza A, Magnette J (2012) Reduced exposure evaluation of an electrically heated cigarette smoking system. Part 7: a 1-month, randomized, ambulatory, controlled clinical study in Poland. Regul Toxicol Pharmacol 64(2 Suppl):S74-S84. https://doi. org/10.1016/j.yrtph.2012.08.006

32. Murphy J, Liu C, McAdam K, Gaça M, Prasad K, Camacho O, McAughey J, Proctor C (2018) Assessment of tobacco heating product THP1.0. Part 9: the placement of a range of next-generation products on an emissions continuum relative to cigarettes via pre-clinical assessment studies. Regul Toxicol Pharmacol 93:92-104. https://doi.org/10.1016/j.yrtph.2017.10.001

33. Evidence review of e-cigarettes and heated tobacco products 2018: executive summary. Public Health England. https://www.gov.uk/ government/publications/e-cigarettes-and-heated-tobacco-produ cts-evidence-review/evidence-review-of-e-cigarettes-and-heate d-tobacco-products-2018-executive-summary. Accessed 11 Jan 2019

34. Murphy J, Gaca M, Lowe F, Minet E, Breheny D, Prasad K, Camacho O, Fearon IM, Liu C, Wright C, McAdam K, Proctor C (2017) Assessing modified risk tobacco and nicotine products: description of the scientific framework and assessment of a closed modular electronic cigarette. Regul Toxicol Pharmacol 90:342357. https://doi.org/10.1016/j.yrtph.2017.09.008

35. Proctor $\mathrm{C}$ et al (2018) Assessment of tobacco heating product THP1.0. Parts 1-9. Regul Toxicol Pharmacol 93:1-104

36. Deveci SE, Deveci F, Acik Y, Ozan AT (2004) The measurement of exhaled carbon monoxide in healthy smokers and non-smokers. Respir Med 98(6):551-556

37. Scherer G, Newland K, Papadopoulou E, Minet E (2014) A correlation study applied to biomarkers of internal and effective dose for acrylonitrile and 4-aminobiphenyl in smokers. Biomarkers 19(4):291-301. https://doi.org/10.3109/1354750X.2014.910271

38. Eaton D, Jakaj B, Forster M, Nicol J, Mavropoulou E, Scott K, Liu C, McAdam K, Murphy J, Proctor CJ (2018) Assessment of tobacco heating product THP1.0. Part 2: product design, operation and thermophysical characterisation. Regul Toxicol Pharmacol 93:4-13. https://doi.org/10.1016/j.yrtph.2017.09.009

39. Fagerström K, Russ C, Yu CR, Yunis C, Foulds J (2012) The Fagerström test for nicotine dependence as a predictor of smoking abstinence: a pooled analysis of varenicline clinical trial data. Nicotine Tob Res 14(12):1467-1473. https://doi.org/10.1093/ntr/ nts018Epub 2012 Mar 30 
40. Olufade AO, Shaw JW, Foster SA, Leischow SJ, Hays RD, Coons SJ (1999) Development of the smoking cessation quality of life questionnaire. Clin Ther 21(12):2113-2130

41. Roux A et al (2010) Early improvement in peripheral vascular tone following smoking cessation using nicotine replacement therapy: aortic wave reflection analysis. Cardiology 117(1):37-43. https:// doi.org/10.1159/000319596

42. O'Brien PC, Fleming TR (1979) A multiple testing procedure for clinical trials. Biometrics 35:549-556

43. Shepperd CJ, Newland N, Eldridge A, Haswell L, Lowe F, Papadopoulou E, Camacho O, Proctor CJ, Graff D, Meyer I (2015) Changes in levels of biomarkers of exposure and biological effect in a controlled study of smokers switched from conventional cigarettes to reduced-toxicant-prototype cigarettes. Regul Toxicol Pharmacol 72(2):273-291. https://doi.org/10.1016/j.yrtph .2015.04.016

44. Scheuermann TS, Richter KP, Rigotti NA, Cummins SE, Harrington KF, Sherman SE, Zhu SH, Tindle HA, Preacher KJ, Consortium of Hospitals Advancing Research on Tobacco (CHART) (2017) Accuracy of self-reported smoking abstinence in clinical trials of hospital-initiated smoking interventions. Addiction 112(12):2227-2236. https://doi.org/10.1111/add.13913

45. Houghton D, Jones TW, Cassidy S, Siervo M, MacGowan GA, Trenell MI, Jakovljevic DG (2016) The effect of age on the relationship between cardiac and vascular function. Mech Ageing Dev 153:1-6. https://doi.org/10.1016/j.mad.2015.11.001

46. Scherer G (2018) Suitability of biomarkers of biological effects (BOBEs) for assessing the likelihood of reducing the tobacco related disease risk by new and innovative tobacco products: a literature review. Regul Toxicol Pharmacol 94:203-233. https:// doi.org/10.1016/j.yrtph.2018.02.002

47. Chobanian AV, Bakris GL, Black HR et al (2003) Seventh report of the Joint National Committee on prevention, detection, evaluation, and treatment of high blood pressure. Hypertension 42(6):1206-1252

48. Higashi Y, Maruhashi T, Noma K et al (2014) Oxidative stress and endothelial dysfunction: clinical evidence and therapeutic implications. Trends Cardiovasc Med 24(4):165-169. https://doi. org/10.1016/j.tcm.2013.12.001

49. Lowe FJ, Luettich K, Talikka M, Hoang V, Haswell LE, Hoeng J, Gaca MD (2017) Development of an adverse outcome pathway for the onset of hypertension by oxidative stress-mediated perturbation of endothelial nitric oxide bioavailability. Appl In Vitro Toxicol 3(1):131-148

50. Safar ME (2018) Arterial stiffness as a risk factor for clinical hypertension. Nat Rev Cardiol 15(2):97-105. https://doi. org/10.1038/nrcardio.2017.155

Publisher's Note Springer Nature remains neutral with regard to jurisdictional claims in published maps and institutional affiliations. 